\title{
Safety, pharmacokinetics and pharmacodynamics of remogliflozin etabonate, a novel SGLT2 inhibitor, and metformin when co-administered in subjects with type 2 diabetes mellitus
}

Elizabeth K Hussey ${ }^{1}$, Anita Kapur ${ }^{1 *}$, Robin O'Connor-Semmes ${ }^{1}$, Wenli Tao ${ }^{1}$, Bryan Rafferty ${ }^{1 \wedge}$, Joseph W Polli ${ }^{1}$, Charles D James $\mathrm{Jr}^{2}$ and Robert $L$ Dobbins ${ }^{1}$

\begin{abstract}
Background: The sodium-dependent glucose co-transporter-2 (SGLT2) is expressed in absorptive epithelia of the renal tubules. Remogliflozin etabonate (RE) is the prodrug of remogliflozin, the active entity that inhibits SGLT2. An inhibitor of this pathway would enhance urinary glucose excretion (UGE), and potentially improve plasma glucose concentrations in diabetic patients. RE is intended for use for the treatment of type 2 diabetes mellitus (T2DM) as monotherapy and in combination with existing therapies. Metformin, a dimethylbiguanide, is an effective oral antihyperglycemic agent widely used for the treatment of T2DM.

Methods: This was a randomized, open-label, repeat-dose, two-sequence, cross-over study in 13 subjects with T2DM. Subjects were randomized to one of two treatment sequences in which they received either metformin alone, RE alone, or both over three, 3-day treatment periods separated by two non-treatment intervals of variable duration. On the evening before each treatment period, subjects were admitted and confined to the clinical site for the duration of the 3-day treatment period. Pharmacokinetic, pharmacodynamic (urine glucose and fasting plasma glucose), and safety (adverse events, vital signs, ECG, clinical laboratory parameters including lactic acid) assessments were performed at check-in and throughout the treatment periods. Pharmacokinetic sampling occurred on Day 3 of each treatment period.

Results: This study demonstrated the lack of effect of RE on steady state metformin pharmacokinetics. Metformin

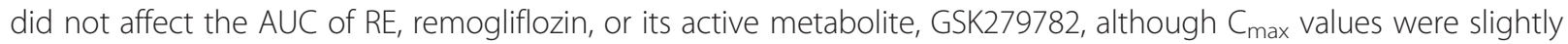
lower for remogliflozin and its metabolite after co-administration with metformin compared with administration of RE alone. Metformin did not alter the pharmacodynamic effects (UGE) of RE. Concomitant administration of metformin and RE was well tolerated with minimal hypoglycemia, no serious adverse events, and no increase in lactic acid.
\end{abstract}

Conclusions: Coadministration of metformin and RE was well tolerated in this study. The results support continued development of RE as a treatment for T2DM.

Trial registration: ClinicalTrials.gov, NCT00376038

Keywords: Remogliflozin etabonate, SGLT2 inhibitor, Metformin, Pharmacokinetics, Type 2 diabetes mellitus

\footnotetext{
* Correspondence: anita.x.kapur@gsk.com

Deceased

'GlaxoSmithKline, 5 Moore Drive, Research Triangle Park, NC 27709, USA

Full list of author information is available at the end of the article
} 


\section{Background}

Type 2 diabetes mellitus (T2DM) is a chronic disease characterized by deteriorating glycemic control and an associated risk of complications. Evidence from controlled clinical trials suggests that improving glycemic control can substantially reduce the long-term microvascular complications of diabetes [1-5]. Current guidelines recommend that T2DM patients should be initially managed with diet and exercise followed by pharmacological treatment with metformin as the preferred step 1 agent, unless there are contraindications to metformin use. When glycemic goals are not achieved, the dose of metformin is increased or a second agent is added [6,7]. In this treatment algorithm, suitability for combination with metformin becomes a critical concern in developing new antidiabetic agents.

Metformin is a dimethylbiguanide that reduces elevated blood glucose levels primarily through its effects on reducing hepatic glucose production and improving peripheral tissue sensitivity to insulin. Metformin is typically administered with meals and has an oral bioavailability of approximately 40 to $60 \%$ [8]. It undergoes extensive renal excretion 3 times the glomerular filtration rate [9] and has a mean plasma elimination half-life between 4.0 and 8.7 hours. There are no clinically relevant metabolic interactions reported with metformin, and it is neither metabolized nor inhibits the metabolism of other drugs [10]. However, there are several transporter related drug interactions, in particular with cationic drugs that have been reported [9]; these typically don't require a dose adjustment. The main adverse event of clinical concern with metformin is lactic acidosis, a potentially life-threatening side effect that may be associated with high plasma concentrations of metformin and renal insufficiency [11-13].

The low-affinity, high-capacity sodium-dependent glucose co-transporter-2 (SGLT2), which is expressed specifically in the renal proximal tubule $[14,15]$, plays a major role in the reabsorption of glucose by the kidney. SGLT2 has recently gained recognition as a potential therapeutic target for reducing hyperglycemia in T2DM, and several selective SGLT2 inhibitors are being evaluated in the clinic [16-22]. In diabetic animal models, pharmacological inhibition of SGLT2 leads to glucosuria followed by normalization of plasma glucose levels and consequent improvement in insulin resistance [23-25]. This mechanism may provide improvements in both fasting and postprandial hyperglycemia without causing weight gain or other dose-limiting side effects observed with other oral antidiabetic approaches [26].

Remogliflozin etabonate is the prodrug of the highly selective and potent SGLT2 inhibitor, remogliflozin. Administration of remogliflozin etabonate has been shown to increase urinary glucose excretion in a dose-dependent manner in mice and rats and to exhibit antidiabetic efficacy in several diabetic rodent models [27]. Remogliflozin is further metabolized to GSK279782, which is an equally potent inhibitor of SGLT2 [28] but circulates at approximately $20 \%$ of the plasma concentrations of remogliflozin; thus GSK279682 is expected to contribute to some of the observed SGLT-2 inhibitor pharmacology. Single oral doses of remogliflozin etabonate up to $1000 \mathrm{mg}$ in healthy subjects and repeated dosing in subjects with T2DM (up to 1000 mg BID for 2 weeks) have been safe and well tolerated $[29,30]$. Remogliflozin etabonate is intended for use in the treatment of T2DM as monotherapy. Given its mechanism of action, it would be a candidate for combination with metformin and other antidiabetic therapies as well. The osmotic diuresis associated with increased urine glucose excretion provides a potential mechanism for pharmacokinetic drug-drug interactions due to the extensive renal clearance of metformin, although treatment with the diuretic hydrochlorothiazide for 2 weeks had no significant effect on the clearance of metformin in subjects with T2DM [31].

This study was designed to evaluate the effect of remogliflozin etabonate on metformin exposure in T2DM subjects. Secondarily, the effect of metformin on steady state plasma concentrations of remogliflozin etabonate, remogliflozin (active entity) and the active metabolite, GSK279782 was evaluated. Three days of dosing (total of 5 doses) was considered adequate to achieve steady-state conditions for both metformin and remogliflozin. Safety problems that might be related to a pharmacokinetic drugdrug interaction were also monitored.

\section{Methods}

This single-center, Phase 1 study was conducted at Medica Sur Hospital and Clinical Foundation Pharma Unit (CIF-BIOTEC), Mexico. This study was approved by the investigational center ethics committee (Hospital Medical Sur Ethics Committee) and was conducted in accordance with Good Clinical Practice and the principles of the Declaration of Helsinki. All subjects provided their written informed consent before study participation. The study was registered at http://clinicaltrials.gov with the identifier NCT00376038.

\section{Subjects}

Male and female subjects (post-menopausal women or premenopausal women with documented hysterectomy or tubal ligation) with documented T2DM ( $\geq 3$ months), ranging in age from 30 to 64 years and with a body mass index of 22 to $35 \mathrm{~kg} / \mathrm{m}^{2}$, were eligible for the study. Enough subjects were to be enrolled to ensure completion of at least 12 evaluable subjects. Pre-study screening included a medical history, physical examination, medical and laboratory evaluations, including 12-lead ECG, and a urinary drug screen. Subjects were required to be free of clinically significant medical and laboratory abnormalities, to have glycosylated hemoglobin (HbA1c) $<10 \%$, and fasting plasma 
glucose $(\mathrm{FPG})<280 \mathrm{mg} / \mathrm{dL}$, and to be controlled by diet alone or metformin. Standard exclusion criteria concerning blood donation, alcohol and drug use, caffeine intake, and participation in other recent investigational drug studies were applied. In addition, subjects were excluded from participation in the study if they required insulin, had received insulin within the past 3 months, or if they had significant renal disease (as manifested by one or more of the following: creatinine clearance $<60 \mathrm{~mL} / \mathrm{min} / 1.73 \mathrm{~m}^{2}$, urine albumin concentration $\geq 300 \mu \mathrm{g} / \mathrm{mg}$ of creatinine, or a spot urine sample with a urine protein/creatinine ratio $>2.5 \mathrm{mg} /$ $\mathrm{mg}$ (a ratio that approximates the common cut off of $3 \mathrm{~g}$ of protein in urine per 24 hours to exclude subjects with nephrotic range proteinuria [32]).

\section{Study design}

The study was a randomized, open-label, repeat-dose, twosequence, cross-over study in subjects with T2DM who were taking metformin or who were drug naive. Before randomization, eligible subjects were stratified on the basis of their pre-entry treatment regimen: metformin or drug naive. Subjects were randomized to receive one of two treatment sequences depicted in Table 1. Each treatment sequence included three treatment regimens $[\mathrm{A}=$ metformin 500 mg every 12 hours (MET BID), B = remogliflozin etabonate $500 \mathrm{mg}$ every 12 hours (RE BID), and $\mathrm{C}=$ metformin $500 \mathrm{mg}$ + remogliflozin etabonate $500 \mathrm{mg}$ every 12 hours (MET + RE)] administered over three 3-day dosing periods that were separated by two non-treatment intervals of variable duration (minimum of 2 days up to a maximum of 15 days). The last dose of drug for each study period was before breakfast on day 3. Metformin was administered as Glucophage (Bristol-Myers Squibb, New York, NY) and subjects were allowed to continue taking metformin during the non-treatment interval between the first and second treatment periods. On the evening before each treatment period, subjects were admitted and confined to the clinical site for the duration of the 3-day treatment period. Pharmacokinetic (PK), pharmacodynamic (PD; urine glucose and FPG), and safety (adverse events, vital signs, ECG, clinical laboratory parameters including lactic acid) assessments were performed at check-in and throughout the treatment periods. For each treatment period, the PK sampling occurred on Day 3.
Subjects were asked to refrain from drinking grapefruit juice or eating grapefruit for at least 3 days before the first dose until collection of the final PK sample for each treatment period. Subjects were to abstain from alcohol or caffeine- or xanthine-containing products from up to 24 hours prior to admission until collection of the final blood/ urine sample. Subjects who smoked had to be able to abstain from use of tobacco products for the 12-hour PK sampling interval. On days $1-3$ of each treatment regimen, while in-house, subjects were fed breakfast, lunch, and dinner as standard meals with identical meals provided on the PK sampling days. Subjects were given $1700 \mathrm{kcal}$ per day, with calories distributed as 55\% carbohydrate, $25 \%$ fat, and $20 \%$ protein. Breakfast was served at approximately $7 \mathrm{am}$ and dinner at approximately $7 \mathrm{pm}$. Subjects were instructed to complete these meals within 30 minutes. Within $15 \mathrm{mi}$ nutes of completing the meal, the study medications were administered with $240 \mathrm{~mL}$ of water per the randomization schedule. Use of the following concomitant medications was allowed if the dosing regimen had been stable for at least 3 months prior to study enrollment: 3-hydroxy-3-methyl-glutaryl-CoA reductase inhibitors, ACE inhibitors, angiotensin receptor blockers, hydrochlorothiazide (dose of $\leq 25 \mathrm{mg}$ /day), calcium channel blockers, alpha or beta blockers, thyroid hormone (only if TSH in normal range), hormone replacement therapy, inhaled and intranasal corticosteroids, antidepressants (SSRIs only) and multivitamins. Low-dose acetaminophen or ibuprofen $(\leq 1.2 \mathrm{~g} /$ day $)$, and any medications prescribed for treatment of adverse events occurring during the study were also allowed. Concomitant medications were not permitted within 4 hours of study drug administration.

\section{Clinical and laboratory monitoring for safety}

For each treatment period, subjects were admitted to the clinical facility on the evening of Day -1 to undergo checkin procedures including a physical examination, 12-lead ECG, vital signs, clinical laboratory tests (chemistry, hematology and urinalysis), lactic acid measurement, fasting blood glucose measurement, alcohol screen, drugs of abuse screen and pregnancy test (if applicable). On each study day morning, a fasting blood sugar measurement was determined by glucose monitor. On Days 1 and 2, vital signs and a 12-lead ECG were recorded. Samples for clinical laboratory measure were also taken on Days 1 and 3.

Table 1 Treatment sequence regimens

\begin{tabular}{cccccc}
\hline $\begin{array}{c}\text { Treatment } \\
\text { sequence }\end{array}$ & $\begin{array}{c}\text { Period 1 } \\
\text { 3 Days }\end{array}$ & $\begin{array}{c}\text { Interval between dosing } \\
\mathbf{2} \text { to } \mathbf{1 5} \text { days }\end{array}$ & $\begin{array}{c}\text { Period 2 } \\
\text { 3 days }\end{array}$ & $\begin{array}{c}\text { Interval between dosing } \\
\text { 2 to 15 days }\end{array}$ & $\begin{array}{c}\text { Period } 3 \\
\mathbf{3} \text { days }\end{array}$ \\
\hline 1 & $\mathrm{~A}$ & Continue metformin only & $\mathrm{C}$ & Stop all trial medications \\
2 & $\mathrm{C}$ & Continue metformin only & $\mathrm{A}$ & Stop all trial medications \\
\hline
\end{tabular}

Treatment A (MET BID): Metformin IR 500 mg every 12 hours. Treatment B (RE BID): Remogliflozin etabonate 500 mg every 12 hours. Treatment C (MET + RE BID): Metformin IR $500 \mathrm{mg}$ every 12 hours + remogliflozin etabonate $500 \mathrm{mg}$ every 12 hours. Metformin was administered starting from the morning of Day 1 of Period 1 and stopped after the morning dose on Day 3 of Period 2. For any Treatment Period when remogliflozin etabonate was administered, remogliflozin etabonate dosing was stopped after the morning dose was given on Day 3. 
Subjects returned to the clinic 7-10 days following the last dosing day for a follow-up physical examination and laboratory evaluation. During the between-treatment intervals, subjects were provided with glucose monitors to measure fasting blood glucose concentrations; subjects were instructed as to how to recognize and treat symptoms of hypoglycemia. Adverse events were monitored throughout the entire study (randomization to follow-up visit). Any adverse events reported during the study were assessed by the investigator for intensity (mild, moderate, severe) and relationship to the study drug (causality). Where possible, all adverse events were followed until stabilization, resolution, or until the event was otherwise explained.

\section{Pharmacokinetic assessment Blood sampling}

Serial blood (two $2 \mathrm{~mL}$ samples for metformin and for remogliflozin etabonate and metabolites) were collected predose, $0.25,0.5,0.75,1,1.5,2,3,4,6,8$, and 12 hours post-dose for determination of plasma metformin, remogliflozin etabonate (prodrug), remogliflozin (active entity) and GSK279782 (metabolite) concentrations. All sample times are relative to the time of the administration of the first dose of study medication on Day 3 of each period. Blood samples for metformin were collected into tubes containing EDTA and immediately placed on ice and centrifuged at approximately $3000 \mathrm{rpm}$ for 10 minutes at approximately $4^{\circ} \mathrm{C}$. The harvested plasma was separated, frozen and stored at $-20^{\circ} \mathrm{C}$ or lower until analysis for metformin concentrations. Blood samples for remogliflozin etabonate, remogliflozin and GSK279782 were collected into tubes containing potassiumoxalate/ sodium fluoride, placed on ice and centrifuged at approximately $3000 \mathrm{rpm}$ for 10 minutes at approximately $4^{\circ} \mathrm{C}$. The harvested plasma was frozen at $-70^{\circ} \mathrm{C}$ until analysis for remogliflozin etabonate, remogliflozin and GSK279782 concentrations.

\section{Drug assays}

The concentrations of remogliflozin etabonate, remogliflozin, and GSK279782 in deproteinized plasma samples and standards were determined by high-performance liquid chromatography (HPLC) with tandem mass spectrometry (MS/ MS) using isotopically labelled internal standards $\left(\left[{ }^{2} \mathrm{H}_{7}\right]-\right.$ remogliflozin etabonate, $\left[{ }^{2} \mathrm{H}_{7}\right]$ - remogliflozin and $\left[{ }^{2} \mathrm{H}_{7}\right]$ GSK279782 as previously described [28].

The concentrations of metformin in plasma were determined by HPLC-MS/MS using a $\left[{ }^{2} \mathrm{H}_{6}\right]$-metformin isotopically labelled internal standard. Plasma proteins from a 50 $\mathrm{mL}$ plasma aliquot were precipitated using acetonitrile containing the internal standard (200 ng mL-1). Samples were vortex mixed then centrifuged. The resulting supernatant was transferred and mixed with $200 \mathrm{~mL}$ of HFBA buffer (water containing $10 \mathrm{mM}$ ammonium acetate and $0.26 \%(\mathrm{v} / \mathrm{v})$ of heptofluorobutyric acid) prior to injection.
HPLC was performed on a Shimadzu LC-10A HPLC system. Chromatography was performed on a MAC-MOD Ace 3 $\mathrm{C} 18,4.6 \times 50 \mathrm{~mm}$ column at a flow rate of $1.0 \mathrm{~mL}$ min- 1 . An isocratic mobile phase elution with 82:18 (v/v) HFBA buffer : Acetonitrile was used. Samples were analysed in positive ion mode by Turbo Ionspray LC/MS/MS with a PE/Sciex API 3000. The calibration range was 20 to 5000 ng mL-1. Performance of the method was assessed during a 3 day validation study using quality control samples at 5 concentrations 20, 80, 500, 4000 and $5000 \mathrm{ng} \mathrm{mL}-1$. The average within-run precision [coefficient of variation (CV \%)] was $<9.6 \%$ and the between-run precision CV\% was $<4.7 \%$. Similar assay performance was observed during study sample analyses.

\section{Pharmacokinetic calculations}

PK analyses of plasma concentration-time data of each analyte (i.e., metformin, remogliflozin etabonate, remogliflozin, and GSK279782) were conducted using the noncompartmental Model 200 (for extravascular administration) of WinNonlin Professional Edition version 4.1 (Pharsight Corporation, Mountain View, CA, USA). Actual elapsed time from dosing was used to estimate all individual plasma PK parameters. Values for the following PK parameters were estimated for each analyte, as appropriate, following administration of 3 days dosing of metformin, remogliflozin etabonate, or both.

- $\mathrm{C}_{\max }$ and $\mathrm{t}_{\max }$ were the actual observed values.

- $\mathrm{AUC}_{(0-12)}$ or $\mathrm{AUC}_{(0-\text { last })}$ was calculated by a combination of linear and logarithmic trapezoidal methods. The linear trapezoidal method was used for all incremental trapezoids arising from increasing concentrations and the logarithmic trapezoidal method was used for those arising from decreasing concentrations.

\section{Pharmacodynamic assessment Plasma PD}

FPG concentrations were collected on Day -1, 1, 2 and 3. Changes in plasma glucose from baseline (Day 1) to Day 2 and 3 were calculated.

\section{Urine PD}

Urine was collected on Days 1 to 3 of each treatment period, and urine glucose concentrations were analyzed for the following intervals: 0-4 hours, 4-8 hours, 8-12 hours and 12-24 hours. The quantity of glucose and creatinine excreted in urine was determined by multiplying the urine glucose or creatinine concentration for each time interval by the volume of urine for the corresponding collection interval. The total 24-hour quantity of glucose excreted in urine on Day 2 was calculated by adding the amounts collected during each interval. Urine glucose and creatinine 
amounts were summarized for each collected interval and for the total 24-hour collection period.

Creatinine clearance (CLcr) was calculated on Day 2 and used to determine the percent of filtered glucose load excreted in urine. By using the urine collections on Day 2, CLcr was calculated as follows:

$$
\begin{aligned}
\mathrm{Clcr}= & \text { total amount of urine creatinine } 0-\mathrm{xxh} \text { interval } \\
& / \text { nearest associated serum creatinine } \\
& =((\text { urine creatinine }(\mathrm{mg} / \mathrm{xx} \text { hours }) \\
& / \text { serum creatinine }(\mathrm{mg} / \mathrm{dL})) * 100 \mathrm{~mL} / \mathrm{dL}) \\
& /\left(\mathrm{xx}^{*} 60\right)(\text { minutes } / \mathrm{xx} \text { hours })
\end{aligned}
$$

where urine creatinine $(\mathrm{mg} / \mathrm{xx}$ hours) is the amount of urine excreted in a xx-hour period. Urine creatinine was calculated by multiplying the urine creatinine concentration by the urine volume $(\mathrm{mL})$ for a 0 -xxh time interval as follows:

$$
\begin{aligned}
& \text { urine creatinine }(\mathrm{mg} / \mathrm{xx} \text { hours }) \\
& =(\text { urine creatinine concentration }(\mathrm{mg} / \mathrm{dL}) * \\
& \quad(\text { interval volume }(\mathrm{mL}) / 100(\mathrm{~mL} / \mathrm{dL}) .
\end{aligned}
$$

CLcr was reported in $\mathrm{mL} /$ minutes on Day 2 for collection intervals $0-4$ hours, $4-8$ hours, $8-12$ hours, $12-$ 24 hours, and the total daily interval of $0-24$ hours.

The serum creatinine concentration used for the above calculations was the pre-dose value for the same day as the urine collection or the one closest to the day of urine collection if no serum creatinine was collected on that day.

\section{Percent of filtered glucose excreted in the urine}

Percent of filtered glucose excreted in the urine was estimated for all collection intervals on Day 2 as follows:

(Glucose Amount Excreted)/(Clcr*PG*Time Interval) or [Urine glucose $(\mathrm{mg} / \mathrm{dL}) *$ Serum Creatinine $(\mathrm{mg} / \mathrm{dL})] /$ [urine creatinine $(\mathrm{mg} / \mathrm{dL}) * \mathrm{PG}(\mathrm{mg} / \mathrm{dL})$ ]

where glucose amount excreted is the amount of glucose excreted during the $\mathrm{xx}$-hour period, and CLcr is calculated for the $\mathrm{xx}$-hour time interval. PG is the plasma glucose concentration reported closest to the midpoint of the time interval. Because only pre-dose PG was collected in this study, the pre-dose PG on Day 2 was used. Time interval is the number of minutes of urine collection for that interval.

Total fluid intake, urine volume, and fluid balance (intake minus output) were summarized over the 0-24-hour interval of Day 1 and Day 2 and the 0-12-hour interval of Day 3 of each treatment period.

\section{Statistical analysis}

The sample size was based on the primary endpoint, metformin $\mathrm{AUCs}_{(0-12)}$ and assumed a within-subject standard deviation of $0.15[33,34]$ for natural log-transformed AUC . Using the two one-sided $t$-test [35] at type I error $\alpha=0.05$ under a crossover design, 12 subjects should provide at least $90 \%$ power to demonstrate lack of an interaction if the ratio of test to reference is truly 1 and the equivalence criteria for the $90 \%$ confidence interval (CI) is $0.8-1.25$.

Safety and PD parameters were summarized using descriptive statistics. Analyses of steady-state plasma metformin $\mathrm{AUC}_{(0-12)}$ and $\mathrm{C}_{\max }$ were conducted with metformin alone as the reference treatment. A mixed effect model with $\ln (\mathrm{AUC}(0-12))$ as the dependent variable; treatment, period and sequence as fixed effects; and subject-within-sequence as a random effect was used to estimate the treatment difference and its associated $90 \% \mathrm{CI}$ on the log scale. The PROC MIXED from SAS (Version 8.2, Cary, NC, USA) was used to fit the model. The estimates and the $90 \% \mathrm{CI}$ were exponentiated in order to obtain the ratio of geometric means and its CI. The assumptions underlying the model were assessed by visual inspection of residual plots.

Similar analyses were performed for the secondary PK endpoints for remogliflozin etabonate, remogliflozin and its metabolite, with and without metformin. $\mathrm{T}_{\max }$ was analysed non-parametrically using Hodges-Lehmann method [36,37].

\section{Results}

Thirteen subjects (7 females [54\%] and 6 males [46\%]) were randomized and completed the study. Of these 13 subjects, 10 subjects were being treated with metformin before study entry and three subjects were drug naive before study entry. The median age was 54 years (range 38 to 62 years); the median BMI was $29 \mathrm{~kg} / \mathrm{m}^{2}$ (range 22.5 to $34.3 \mathrm{~kg} / \mathrm{m}^{2}$ ); mean fasting plasma glucose at baseline was $7.21 \mathrm{mmol} / \mathrm{L}$ (SD 1.77; range 4.8 to $10.9 \mathrm{mmol} / \mathrm{L}$ ). All subjects were Hispanic or Latino.

\section{Pharmacokinetics}

The summary data of PK parameters for metformin, remogliflozin etabonate, remogliflozin and GSK279782 are presented in Table 2. The primary PK objective was to demonstrate a lack of effect of remogliflozin etabonate on the PK parameters of metformin. Results from the primary comparison, are summarized in Table 3 and mean concentration vs time profiles are shown in Figure 1. There was no effect of remogliflozin etabonate on metformin PK parameters.

One of the secondary objectives included a comparison of PK parameters for remogliflozin etabonate, remogliflozin and GSK279782 after treatment with remogliflozin etabonate alone and with MET + RE. A summary of these 
Table 2 Summary of plasma metformin, remogliflozin etabonate, remogliflozin, and GSK279782 PK parameters

\begin{tabular}{|c|c|c|}
\hline Metformin PK parameter & MET BID & MET + RE BID \\
\hline$A \cup C_{(0-12)}(h \cdot n g / m L)$ & $\begin{array}{c}\mathbf{N}=13 \\
7141.3(24)\end{array}$ & $\begin{array}{c}\mathbf{N}=\mathbf{1 3} \\
7520.8(27)\end{array}$ \\
\hline$C_{\max }(n g / m L)$ & $1018.2(26)$ & $1025.3(25)$ \\
\hline$t_{\max }(h)$ & $4.0(1.0-6.0)$ & $4.0(1.0-6.0)$ \\
\hline \multirow[t]{2}{*}{ Remogliflozin etabonate (prodrug) PK Parameter } & RE BID & MET + RE BID \\
\hline & $N=12-13^{a}$ & $N=12-13^{a}$ \\
\hline$A \cup C_{(0-\text { last }}(\mathrm{h} \cdot \mathrm{ng} / \mathrm{mL})$ & $98.9(69)$ & $102.1(49)$ \\
\hline$C_{\max }(\mathrm{ng} / \mathrm{mL})$ & $79.5(107)$ & $67.7(77)$ \\
\hline$t_{\max }(h)$ & $3.0(1.0-4.0)$ & $3.0(1.0-6.0)$ \\
\hline \multirow[t]{2}{*}{ Remogliflozin (active entity) PK Parameter } & RE BID & $\mathrm{MET}+\mathrm{RE}$ BID \\
\hline & $\mathrm{N}=13$ & $N=13$ \\
\hline$A \cup C_{(0-12)}(h \cdot n g / m L)$ & $6814.3(33)$ & $6425.9(33)$ \\
\hline$C_{\max }(\mathrm{ng} / \mathrm{mL})$ & $2688.6(52)$ & $2124.6(63)$ \\
\hline$t_{\max }(h)$ & $3.0(1.0-4.0)$ & $3.0(1.0-6.0)$ \\
\hline \multirow[t]{2}{*}{ GSK279782 (active metabolite) PK Parameter } & RE BID & $\mathrm{MET}+\mathrm{RE}$ BID \\
\hline & $N=13$ & $N=13$ \\
\hline$A \cup C_{(0-12)}(h \cdot n g / m L)$ & $1527.9(37)$ & $1472.9(36)$ \\
\hline$C_{\max }(n g / m L)$ & $462.8(39)$ & 361.9 (38) \\
\hline$t_{\max }(h)$ & $4.0(1.0-4.0)$ & $4.0(1.0-8.0)$ \\
\hline
\end{tabular}

Values are geometric mean (\%CVb) for each parameter, except for $t_{\max }$ which is median (range). PK, pharmacokinetic; MET BID, metformin $500 \mathrm{mg}$ every 12 hours; $\mathrm{MET}+\mathrm{RE}$ BID, metformin $500 \mathrm{mg}$ + remogliflozin etabonate $500 \mathrm{mg}$ every 12 hours; RE BID, remogliflozin etabonate $500 \mathrm{mg}$ every 12 hours.

${ }^{a}$ AUC not evaluable for one subject.

results is presented in Table 3 and concentration vs. time profiles are provided in Figures 2, 3, 4. There were no effects of metformin on the AUC of remogliflozin etabonate, remogliflozin, or its metabolite, GSK279782. However, $\mathrm{C}_{\max }$ was lower with the combination. For $\mathrm{C}_{\max }$, on average, there was a decrease of $21 \%$ in remogliflozin and a decrease of $22 \%$ in GSK279782 with MET + RE compared to remogliflozin etabonate alone. The $90 \% \mathrm{CI}$ indicates that the true difference lies between a decrease of $40 \%$ and an increase of $5 \%$ for remogliflozin and between a decrease of $33 \%$ and 9\% for GSK279782.

\section{Pharmacodynamics}

\section{Fasting plasma glucose}

A summary of the FPG concentration data by treatment period and study day is presented in Figure 5. When the changes in fasting plasma glucose concentrations from baseline (pre-dose on Day 1) to Day 2 and Day 3 were considered for the three treatment periods, it appeared that the fasting glucose concentrations remained relatively stable during the MET BID period, whereas small decreases were observed during both the RE BID and MET + RE BID treatment periods.

Table 3 Statistical comparisons of PK parameters of metformin, remogliflozin etabonate, remogliflozin, and GSK279782 with and without remogliflozin etabonate

\begin{tabular}{|c|c|c|c|c|}
\hline Compound & PK parameter & Treatment comparison & Point estimate (GLSM Ratio) & $90 \% \mathrm{Cl}$ \\
\hline \multirow[t]{2}{*}{ Metformin } & $A \cup C(0-12)^{[1]}$ & $\mathrm{MET}+\mathrm{RE} / \mathrm{MET}$ & 1.05 & $(0.98,1.12)$ \\
\hline & Cmax & $\mathrm{MET}+\mathrm{RE} / \mathrm{MET}$ & 1.01 & $(0.92,1.10)$ \\
\hline \multirow[t]{2}{*}{ Remogliflozin etabonate (prodrug) } & $A \cup C_{(0-\text { last })}$ & $\mathrm{MET}+\mathrm{RE} / \mathrm{RE}$ & 1.00 & $(0.77,1.29)$ \\
\hline & $C_{\max }$ & $\mathrm{MET}+\mathrm{RE} / \mathrm{RE}$ & 0.85 & $(0.54,1.35)$ \\
\hline \multirow[t]{2}{*}{ Remogliflozin (active entity) } & $A \cup C_{(0-12)}$ & $\mathrm{MET}+\mathrm{RE} / \mathrm{RE}$ & 0.94 & $(0.86,1.04)$ \\
\hline & $C_{\max }$ & $\mathrm{MET}+\mathrm{RE} / \mathrm{RE}$ & 0.79 & $(0.60,1.05)$ \\
\hline \multirow[t]{2}{*}{ GSK279782 (active metabolite) } & $A \cup C_{(0-12)}$ & $\mathrm{MET}+\mathrm{RE} / \mathrm{RE}$ & 0.96 & $(0.92,1.01)$ \\
\hline & $C_{\max }$ & $\mathrm{MET}+\mathrm{RE} / \mathrm{RE}$ & 0.78 & $(0.67,0.91)$ \\
\hline
\end{tabular}

${ }^{[1]}$ primary comparison; MET + RE, metformin $500 \mathrm{mg}+$ remogliflozin etabonate $500 \mathrm{mg}$ every 12 hours; GLSM : Geometric least-squares mean. 


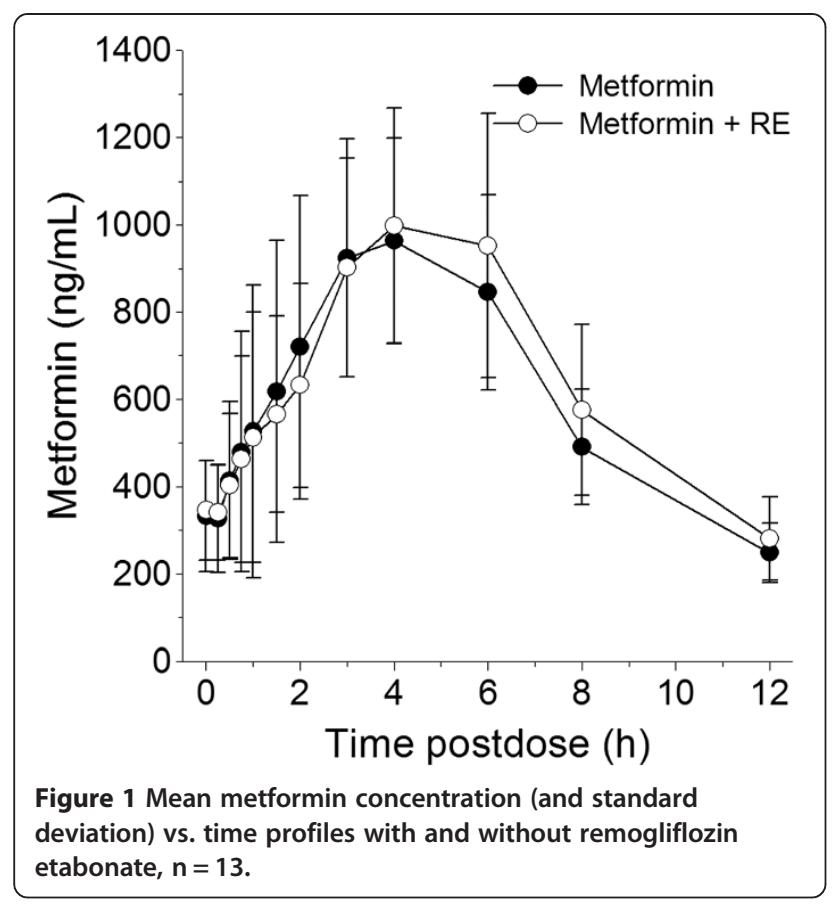

Urinary glucose excretion and percent of filtered glucose excreted

Mean cumulative 24-hour urinary glucose excretion was approximately $500 \mathrm{mmol}$ following treatment with $\mathrm{RE}$ BID or MET + RE BID (Day 2), whereas MET BID had relatively no effect on urine glucose output (Table 4).

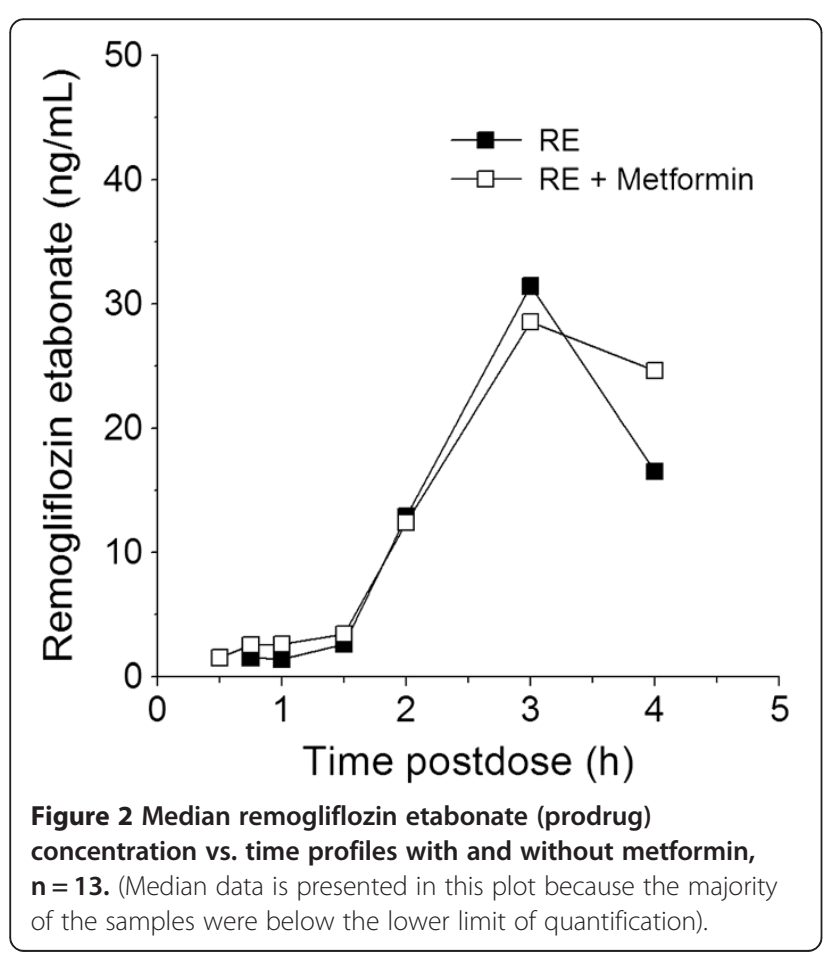

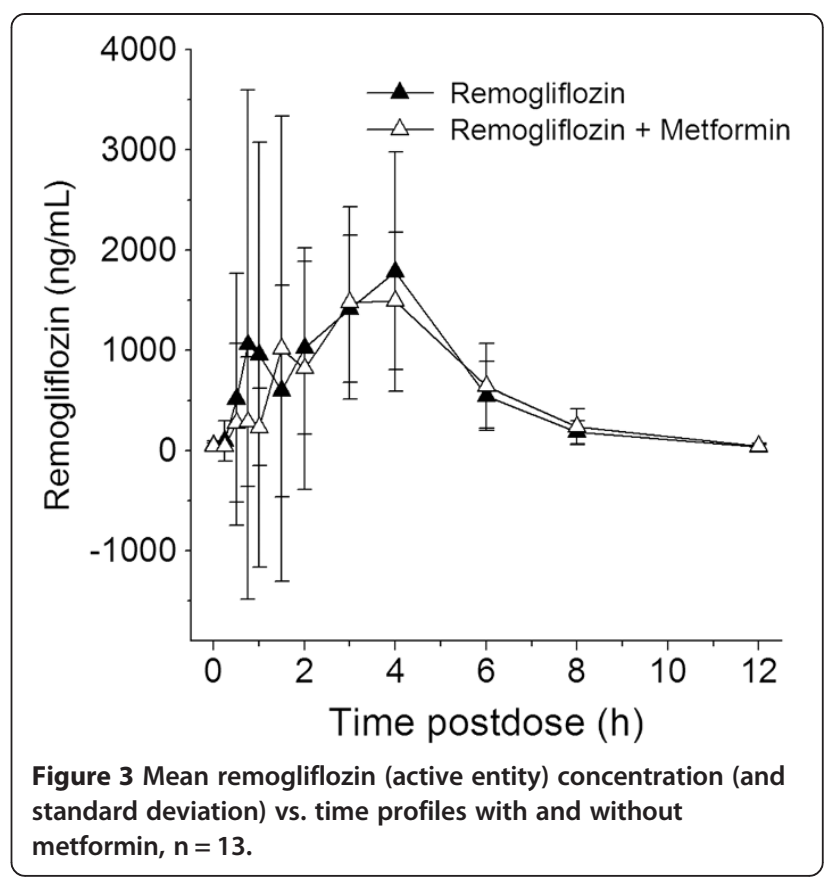

The effect of remogliflozin etabonate on urine glucose excretion was not diminished by co-administration with metformin. The greatest increase in urine glucose excretion was evident within the first 4 hours of dosing following both remogliflozin etabonate regimens. The 24-hour creatinine clearance on Day 2 was comparable across the three treatment periods and was approximately $110 \mathrm{~mL} / \mathrm{min}$. During the RE BID and MET + RE BID periods, mean and median values for the percent

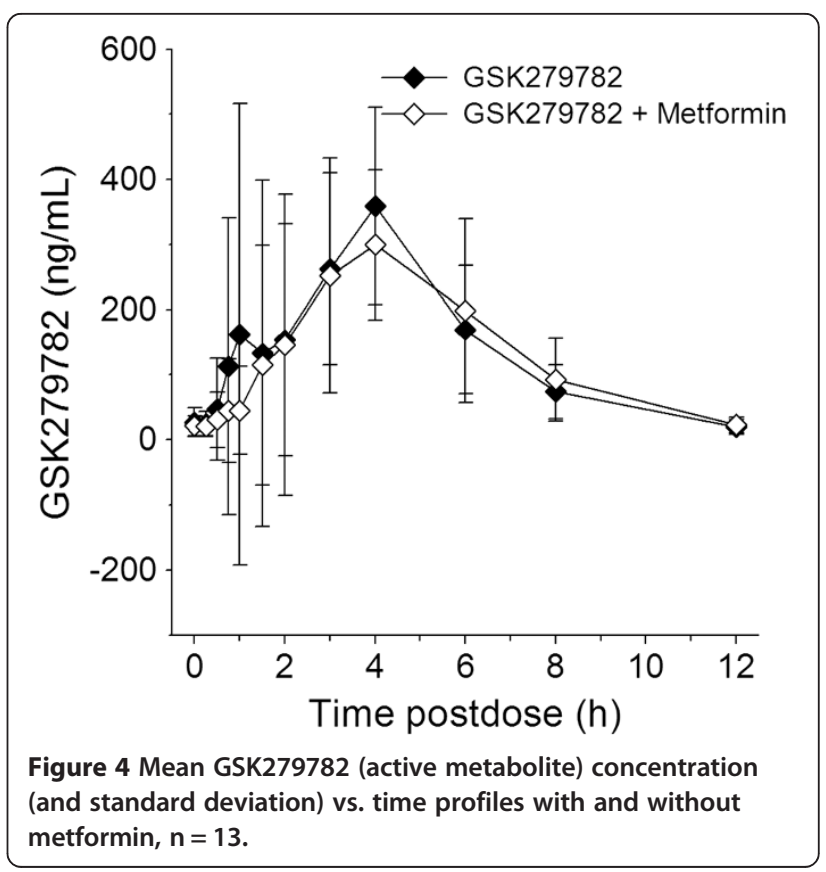




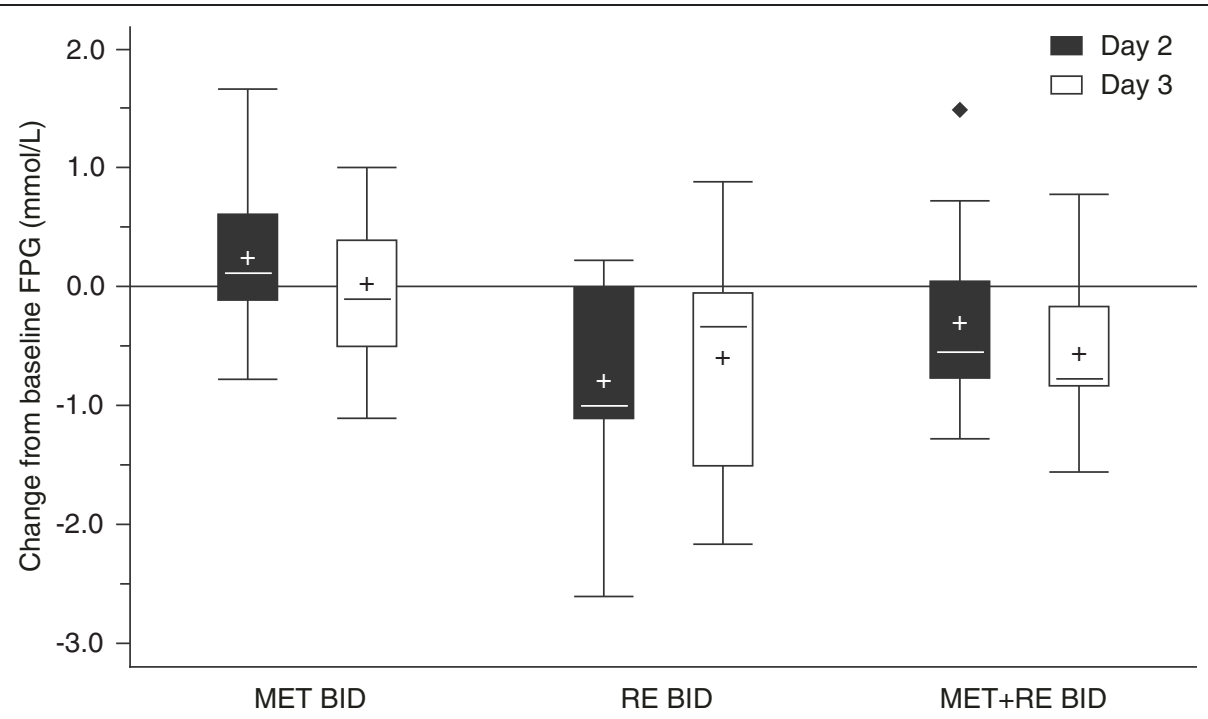

Figure 5 Fasting plasma glucose concentration (FPG; mmol/L) - Change from baseline (pre- dose on Day 1 of each treatment period). MET BID, metformin 500 mg every 12 hours; RE BID, remogliflozin etabonate 500 mg every 12 hours; MET + RE BID, metformin 500 mg + remogliflozin etabonate $500 \mathrm{mg}$ every 12 hours. Mean (and standard deviation) baseline FPG values for each treatment period: MET BID: 6.72 (1.88); RE BID, 6.98 (2.06); MET + RE BID, 6.42 (1.15).

of filtered glucose excreted in the urine ranged from $43 \%$ up to $68 \%$ during the individual collection intervals, with a mean of approximately $50 \%$ for the combined 24 hour collection for both remogliflozin etabonate containing regimens compared to $1.4 \%$ with metformin alone (Table 5).

\section{Fluid balance}

Total fluid intake, total urine volume, and fluid balance data for the 24-hour collection intervals on Days 1 and 2 and the initial 12-hour collection interval on Day 3 were compared by treatment. On Days 1 and 2, mean total 24-hour fluid intake ranged from approximately $2500 \mathrm{~mL}$ to $3000 \mathrm{~mL}$ across the three treatment periods. During the 12-hour collection period on Day 3, mean fluid intake ranged from approximately 1800 to $2200 \mathrm{~mL}$ for any one treatment period. Because fluid intake was less than total urine volume throughout all treatment periods, mean fluid balance values were considered negative during most intervals. On Day 1, fluid balance (median, range) appeared more negative on RE BID (-1145 mL, -1630 to $+335 \mathrm{~mL})$

Table 4 Summary of 24-hour urine glucose ( $\mathrm{mmol}$ ) on day 2 by treatment

\begin{tabular}{lccc}
\hline & Met BID & RE BID & Met + RE BID \\
& $\mathbf{N}=\mathbf{1 3}$ & $\mathbf{N}=\mathbf{1 3}$ & $\mathbf{N}=\mathbf{1 3}$ \\
\hline Mean (SD) & $13.6(13.4)$ & $528(130)$ & $458(98)$ \\
Median & 10.9 & 497 & 485 \\
Min, Max & $1.1,43.9$ & 384,796 & 242,573 \\
\hline
\end{tabular}

and MET + RE BID (-1200 mL, -2395 to $-90 \mathrm{~mL})$ compared to MET BID $(-775 \mathrm{~mL},-2280$ to $+400 \mathrm{~mL})$. Fluid balance neutrality seemed to be reached on Day 3 for all drug regimens.

\section{Safety and tolerability}

There were no serious adverse events reported. The only adverse event considered related to study drug was hypoglycemic symptoms reported by 2 subjects, one event with metformin alone and one with MET + RE. However, plasma glucose measurements were unfortunately not performed to confirm hypoglycemia. In both cases, the symptoms of hypoglycemia were considered mild in intensity. The events were reported in the time before scheduled meals; the symptoms resolved with provision of food, and did not require a change in study drug. Back pain and headache were the only events reported by more than one subject during any treatment period (reported during MET BID by 2 different subjects). All adverse events are summarized in Table 6.

Table 5 Summary of percent filtered glucose excreted in urine on day 2 by treatment

\begin{tabular}{lccc}
\hline & Met BID & RE BID & Met + RE BID \\
& $\mathbf{N}=\mathbf{1 3}$ & $\mathbf{N}=\mathbf{1 3}$ & $\mathbf{N}=\mathbf{1 3}$ \\
\hline Mean (SD) & $1.41(1.52)$ & $51.3(7.02)$ & $48.7(9.87)$ \\
Median & 0.95 & 51.8 & 49.3 \\
Min, Max & $0.10,4.71$ & $38.4,61.3$ & $35.7,67.9$ \\
\hline
\end{tabular}


Table 6 Summary of adverse events by treatment

\begin{tabular}{lccc}
\hline Preferred term & MET BID & RE BID & MET + RE BID \\
& $\mathbf{N}=\mathbf{1 3}$ & $\mathbf{N}=\mathbf{1 3}$ & $\mathbf{N}=\mathbf{1 3}$ \\
& $\mathbf{n}(\%)$ & $\mathbf{n}(\%)$ & $\mathbf{n}(\%)$ \\
\hline Any Event & $\mathbf{5 ( 3 8 \% )}$ & $\mathbf{2}(\mathbf{1 5 \% )}$ & $\mathbf{7}(\mathbf{5 4 \% )}$ \\
Headache & $2(15 \%)$ & 0 & $1(8 \%)$ \\
Back pain & $2(15 \%)$ & 0 & 0 \\
Muscle spasms & $1(8 \%)$ & $1(8 \%)$ & 0 \\
Hypoglycemia & $1(8 \%)$ & 0 & $1(8 \%)$ \\
Neck pain & $1(8 \%)$ & 0 & 0 \\
Osteoarthritis & 0 & 0 & $1(8 \%)$ \\
Abdominal pain upper & 0 & $1(8 \%)$ & 0 \\
Dyspepsia & 0 & 0 & $1(8 \%)$ \\
Toothache & $1(8 \%)$ & 0 & 0 \\
Dizziness & $1(8 \%)$ & 0 & 0 \\
Fatigue & $1(8 \%)$ & 0 & 0 \\
Nasopharyngitis & 0 & 0 & $1(8 \%)$ \\
Wound & 0 & 0 & $1(8 \%)$ \\
Rash & 0 & 0 & $1(8 \%)$ \\
\hline
\end{tabular}

No clinically significant changes in laboratory parameters or vital signs were reported for any treatment regimen. As an increased exposure to metformin can result in lactic acidosis, lactic acid levels were measured. While there were no instances of lactic acidosis, a trend toward increasing lactic acid was observed with metformin monotherapy relative to regimens including remogliflozin (Figure 6).

\section{Discussion}

Despite the availability of multiple classes and combinations of antihyperglycemic agents, the clinical management of T2DM is currently suboptimal, with the majority of patients failing to achieve and maintain target glycemic levels in practice [38]. Consequently, there is a continued need for novel therapeutic approaches, particularly those with complementary modes of action that will enable further improvement of glycemic control.

Remogliflozin etabonate, by inhibiting glucose reabsorption, offers a potential treatment for T2DM as monotherapy and in combination with existing therapies. Remogliflozin etabonate is being developed for use for the treatment of T2DM as monotherapy, and in combination with existing therapies including metformin. In this study, no effect of remogliflozin etabonate on metformin PK parameters was observed. The findings from this study are consistent with the reported lack of inhibition by remogliflozin etabonate, remogliflozin, and GSK279782 on a panel of metabolic enzymes and transporters, including organic cation transporters involved with metformin renal secretion [39].

This study was not adequately powered to test the effect of metformin on remogliflozin etabonate PK parameters. Metformin did not appear to affect the AUC of remogliflozin etabonate, remogliflozin and its metabolite; however, $\mathrm{C}_{\max }$ was lower after the co-administration of remogliflozin etabonate and metformin than with remogliflozin etabonate alone. Under the conditions of this study, the peak plasma concentration of remogliflozin considerably exceeded the concentration required for full

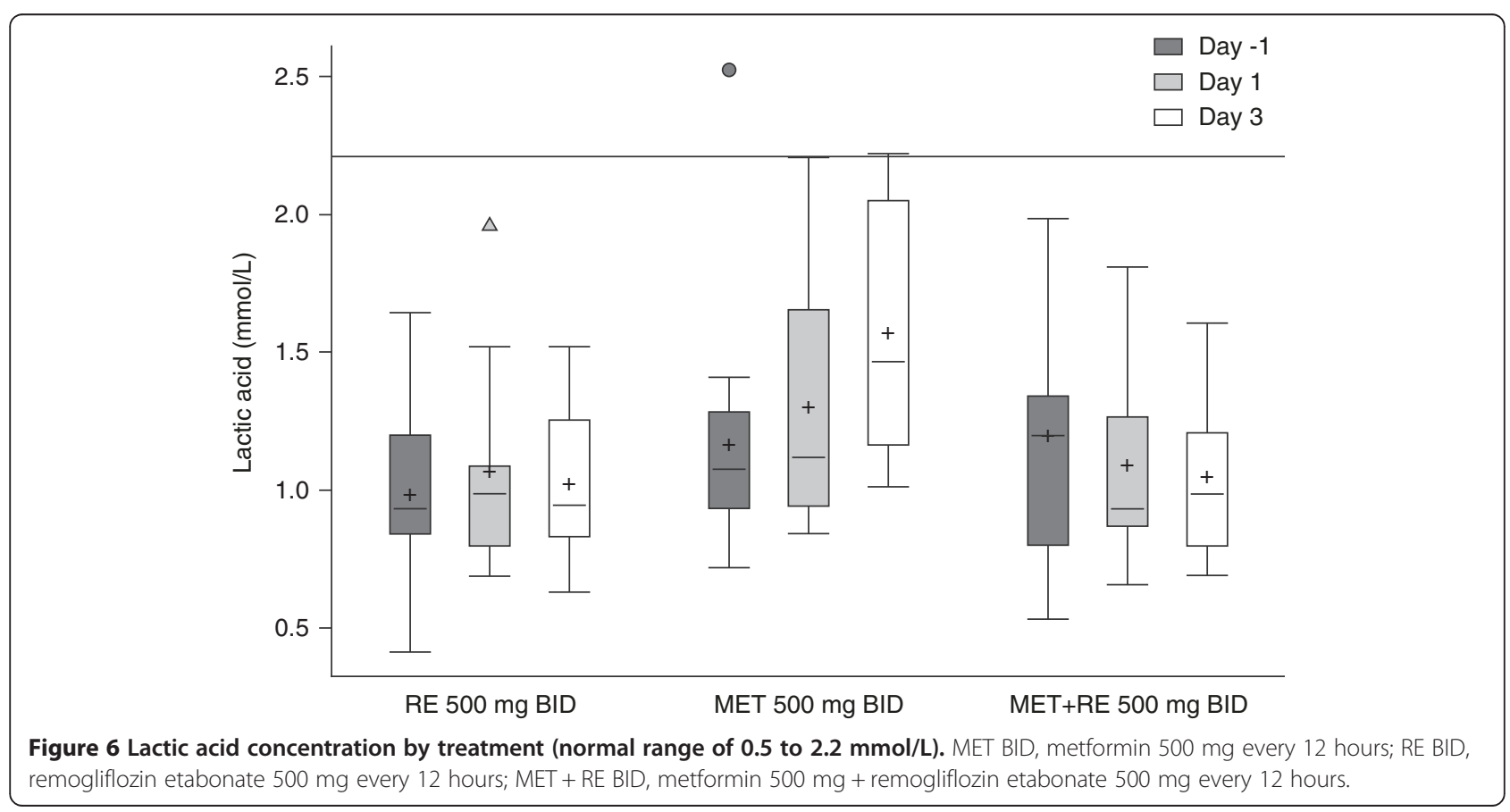


inhibition of the SGLT2 transporter. However, it is possible that a clinically significant decrease would be observed when administering the combination if low doses of remogliflozin etabonate or considerably higher doses of metformin were given.

As expected on the basis of its pharmacological properties, the administration of remogliflozin etabonate with or without metformin greatly increased urine glucose excretion and the percent of filtered glucose excreted in the urine. The evidence of pharmacological effect was seen within the first 4 hours of dosing with remogliflozin etabonate and sustained while on treatment. Co-administration of metformin with remogliflozin etabonate did not diminish the glucosuric effect of remogliflozin etabonate. Only small changes in fasting glucose concentration were observed during both the RE BID and MET + RE BID treatment periods for this cohort of subjects with good glucose control. Mean fasting glucose concentrations were $<7 \mathrm{mmol} / \mathrm{L}$ on Day -1 of each treatment period, leaving little room for substantial improvement.

Concomitant administration of remogliflozin etabonate with metformin for 3 days was well tolerated in subjects with T2DM. Hypoglycemia was the only adverse event that was considered related to study drug (and occurred with metformin alone, as well as with the combination). However, neither case was confirmed with plasma glucose concentrations. Antidiabetic treatments that increase urine glucose may increase risk of urinary tract infections (UTIs); however, no documented UTIs were observed over the limited duration of remogliflozin etabonate treatment in this study. Mean lactate concentrations showed an increase or increasing trend during the three day MET BID treatment period. In contrast, mean lactate concentrations are unchanged or decreased slightly during RE BID and MET + RE BID periods. Potential mechanisms to explain the decreased lactate concentrations include reduced glucose concentrations with less production from glycolysis, enhanced extraction of lactic acid by the liver for gluconeogenesis or increased clearance of lactic acid by the kidney. No symptoms suggestive of lactic acidosis occurred during the study.

\section{Conclusions}

In summary, the findings of this study do not indicate a safety concern when multiple oral doses of remogliflozin etabonate $500 \mathrm{mg}$ are administered with metformin 500 mg BID in the intended patient population. Because remogliflozin etabonate does not affect the PK profile of metformin, there is a low risk for adverse events resulting from a PK drug interaction and increased metformin exposure. The approximate $20 \%$ decline in remogliflozin Cmax under conditions of coadministration is likely a reflection of the $15 \%$ decline in the Cmax of the prodrug
(RE) when given with metformin (Table 3). It appears that metformin reduces the Cmax of RE without an effect on RE AUC, suggesting a change in the shape of the 12-hour, steady state, concentration-time profile. Even though the confidence interval is wide for the prodrug Cmax point estimate $(0.54,1.35)$ and contains 1.0 , it is plausible that coadministration of metformin altered GI motility enough to affect the absorption or hydrolysis of RE resulting in a lower Cmax of RE. The lower Cmax values for remogliflozin and GKS279782 following dosing with metformin collectively support this conclusion since they are downstream metabolites of RE.

Although administration with metformin resulted in a $21 \%$ reduction in $C_{\max }$, the $\mathrm{PD}$ properties of remogliflozin etabonate were not altered when administered with metformin. There was an indication that remogliflozin etabonate alone improves plasma blood glucose by increasing the excretion of urine glucose, and this effect by remogliflozin etabonate was not impaired by the co-administration of metformin. Future studies in a larger patient population are warranted to definitively test the safety and efficacy of remogliflozin etabonate in combination with metformin in patients with T2DM who have not achieved the desired glycemic target.

\section{Competing interests}

At the time of study, EKH, AK, ROCS, WT, BR, JWP, CJ, and RLD are employees of GlaxoSmithKline.

\section{Authors' contributions}

EKH, AK, ROCS, WT, BR, JWP, CJ, and RLD participated in the design of the study, its co-ordination and performed the statistical analysis. All authors were involved in critically revising the drafts of the manuscript, and read and approved the final manuscript.

\section{Acknowledgements}

The authors would like to acknowledge Drs. Jorge Poo and Esteban Rios, clinical investigators (Medica Sur Hospital and Clinical Foundation, CIFBIOTEC, Mexico City, Mexico) for their efforts in conduct of this clinical study. Editorial assistance in the preparation of this manuscript was provided by Katie Green, International Medical Press, funded by GlaxoSmithKline.

\section{Author details}

'GlaxoSmithKline, 5 Moore Drive, Research Triangle Park, NC 27709, USA. ${ }^{2}$ Tandem Labs, Durham, NC, USA.

Received: 17 February 2012 Accepted: 18 April 2013 Published: 30 April 2013

\section{References}

1. DCCT Research Group: The relationship of glycemic exposure (HbA1c) to the risk of development and progression of retinopathy in the Diabetes Control and Complications Trial. Diabetes 1995, 44:968-983.

2. Ohkubo Y, Kishikawa H, Araki E, Miyata T, Isami S, Motoyoshi S, Kojima Y, Furuyoshi N, Shichiri M: Intensive insulin therapy prevents the progression of diabetic microvascular complications in Japanese patients with non-insulin-dependent diabetes mellitus: a randomized prospective 6-year study. Diabetes Res Clin Pract 1995, 28:103-117.

3. Stratton IM, Adler Al, Neil HA, Matthews DR, Manley SE, Cull CA, Hadden D, Turner RC, Holman RR: Association of glycaemia with macrovascular and microvascular complications of type 2 diabetes (UKPDS 35): prospective observational study. Br Med J 2000, 321:405-412. 
4. UK Prospective Diabetes Study (UKPDS) Group: Effect of intensive bloodglucose control with metformin on complications in overweight patients with type 2 diabetes (UKPDS 34). Lancet 1998, 352:854-865.

5. UK Prospective Diabetes Study (UKPDS) Group: Intensive blood-glucose control with sulphonylureas or insulin compared with conventional treatment and risk of complications in patients with type 2 diabetes (UKPDS 33). Lancet 1998, 352:837-853.

6. American Diabetes Association: Standards of medical care in diabetes. In PhD Thesis; 2007.

7. Nathan D, Buse J, Davidson M, Heine R, Holman R, Sherwin R, Zinman B: Management of hyperglycaemia in type 2 diabetes: a consensus algorithm for the initiation and adjustment of therapy. Diabetologia 2006, 49:1711-1721

8. Scheen AJ: Clinical pharmacokinetics of metformin. Clin Pharmacokinet 1996, 30:359-371.

9. Graham GG, Punt J, Arora M, Day R, Doogue MP, Duopng JK, Furlong TJ, Greenfield JR, Greenup LC, Kirkpatrick CM, Ray JE, Timmins P, Williams KM: Clinical pharmacokinetics of metformin. Clin Pharmacokinet 2011, 50:81-98.

10. Scheen AJ: Drug interactions of clinical importance with antihyperglycaemic agents: an update. Drug Saf 2005, 28:601-631.

11. Bodmer M, Meier C, Krahenbuhl S, Jick SS, Meier CR: Metformin, sulfonylureas, or other antidiabetes drugs and the risk of lactic acidosis or hypoglycemia: a nested case-control analysis. Diabetes Care 2008, 31:2086-2091

12. Davis TM, Jackson D, Davis WA, Bruce DG, Chubb P: The relationship between metformin therapy and the fasting plasma lactate in type 2 diabetes: the fremantle diabetes study. Br J Clin Pharmacol 2001, 52:137-144.

13. Salpeter SR, Greyber E, Pasternak GA, Salpeter EE: Risk of fatal and nonfatal lactic acidosis with metformin use in type 2 diabetes mellitus: systematic review and meta-analysis. Arch Intern Med 2003, 163:2594-2602.

14. Kanai Y, Lee WS, You G, Brown D, Hediger MA: The human kidney low affinity $\mathrm{Na}+/$ glucose cotransporter SGLT2. Delineation of the major renal reabsorptive mechanism for D-glucose. J Clin Invest 1994, 93:397-404.

15. Wright EM, Hirayama BA, Loo DF: Active sugar transport in health and disease. J Intern Med 2007, 261:32-43.

16. Handlon AL: Sodium glucose co-transporter 2 (SGLT2) inhibitors as potential antidiabetic agents. Expert Opin Ther Pat 2005, 13:1531-1540.

17. Hussey E, Clark R, Amin D, Kipnes M, O'Connor-Semmes R, O'Driscoll E, Leong J, Murray S, Dobbins R, Layko D, et al: The single-dose pharmacokinetics and pharmacodynamics of sergliflozin etabonate, a novel inhibitor of glucose reabsorption, in healthy volunteers and subjects with type 2 diabetes mellitus. J Clin Pharmacol 2010, 50:623-635.

18. Hussey EK, Dobbins RL, Stoltz RR, Stockman NL, O'Connor-Semmes RL, Kapur A, Murray SC, Layko D, Nunez DJR: Multiple-dose pharmacokinetics and pharmacodynamics of sergliflozin etabonate, a novel inhibitor of glucose reabsorption, in healthy overweight and obese subjects: a randomized double-blind study. J Clin Pharmacol 2010, 50:636-646.

19. Idris I, Donnelly R: Sodium-glucose co-transporter-2 inhibitors: an emerging new class of oral antidiabetic drug. Diabetes Obes Metab 2009, 11:79-88.

20. Isaji M: Sodium-glucose cotransporter inhibitors for diabetes. Curr Opin Investig Drugs 2007, 8:285-292.

21. Komoroski B, Vachharajani N, Feng Y, Li L, Kornhauser D, Pfister M: Dapagliflozin, a novel, selective SGLT2 inhibitor, improved glycemic control over 2 weeks in patients with type 2 diabetes mellitus. Clin Pharmacol Ther 2009, 85:513-519.

22. Komoroski B, Vachharajani N, Boulton D, Kornhauser D, Geraldes M, Li L, Pfister M: Dapagliflozin, a novel SGLT2 inhibitor, induces dosedependent glucosuria in healthy subjects. Clin Pharmacol Ther 2009, 85:520-526.

23. Asano T, Ogihara T, Katagiri $H$, Sakoda H, Ono H, Fujishiro M, Anai M, Kurihara $\mathrm{H}$, Uchijima Y: Glucose transporter and $\mathrm{Nat}$ /glucose cotransporter as molecular targets of anti-diabetic drugs. Curr Med Chem 2004, 11:2717-2724.

24. Ehrenkranz JR, Lewis NG, Kahn CR, Roth J: Phlorizin: a review. Diabetes Metab Res Rev 2005, 21:31-38.

25. Katsuno K, Fujimori Y, Takemura Y, Hiratochi M, Itoh F, Komatsu Y, Fujikura H, Isaji M: Sergliflozin, a novel selective inhibitor of low-affinity sodium glucose cotransporter (SGLT2), validates the critical role of SGLT2 in renal glucose reabsorption and modulates plasma glucose level. J Pharmacol Exp Ther 2007, 320:323-330.
26. Jabbour SA, Goldstein BJ: Sodium glucose co-transporter 2 inhibitors: blocking renal tubular reabsorption of glucose to improve glycaemic control in patients with diabetes. Int J Clin Pract 2008, 62:1279-1284.

27. Fujimori Y, Katsuno K, Nakashima I, Ishikawa-Takemura Y, Fujikura H, Isaji M Remogliflozin etabonate, in a novel category of selective low-affinity sodium glucose cotransporter (SGLT2) inhibitors, exhibits antidiabetic efficacy in rodent models. J Pharmacol Exp Ther 2008, 327:268-276.

28. Sigafoos J, Bowers G, Castellino S, Culp AG, Wagner DS, Reese JM, Humphreys JE, Hussey EK, O'Connor-Semmes RL, Kapur A, Tao W, Dobbins RL, Polli JW: Assessment of the drug interaction risk for remogliflozin etabonate, a sodium-dependent glucose cotransporter-2 inhibitor: evidence from in vitro, human mass balance, and ketoconazole interaction studies. Drug Metab Dispos 2012, 40:2090-2101.

29. Dobbins R, Kapur A, Kapitza C, O'Connor-Semmes R, Tao W, Hussey E: Remogliflozin etabonate, a selective inhibitor of the sodium-glucose transporter 2 (SCLT2) reduces serum glucose in type 2 diabetes mellitus (T2DM) patients. Diabetes 2009, 58:1573-P.

30. Kapur A, O'Connor-Semmes R, Hussey E, Dobbins R, Tao W, Hompesch M, Nunez D: First human dose escalation study with remogliflozin etabonate (RE) in healthy subjects and in subjects with type 2 diabetes mellitus (T2DM). Diabetes 2009, 58:509-P.

31. Sung EY, Moore MP, Lunt H, Doogue M, Zhang M, Begg EJ: Do thiazide diuretics alter the pharmacokinetics of metformin in patients with type 2 diabetes already established on metformin? Br J Clin Pharmacol 2009, 67:130-131.

32. Thurman J, Wiseman A: The patient with glomerulonephritis or vasculitis In Manual of Nephrology. 7th edition. Edited by Schrier RW. Philadelphia: Lippincott Williams \& Wilkins; 2009:140-153.

33. Saffar F, Aiache JM, Andre P: Influence of food on the disposition of the antidiabetic drug metformin in diabetic patients at steady-state. Methods Find Exp Clin Pharmacol 1995, 17:483-487.

34. Timmins P, Donahue S, Meeker J, Marathe P: Steady-state pharmacokinetics of a novel extended-release metformin formulation. Clin Pharmacokinet 2005, 44:721-729.

35. Schuirmann DJ: A comparison of the two one-sided tests procedure and the power approach for assessing the equivalence of average bioavailability. J Pharmacokinet Biopharm 1987, 15:657-680.

36. Hauschke D, Steinijans WW, Diletti E: A distribution-free procedure for the statistical analysis of bioequivalence studies. Int $J$ Clin Pharmacol Ther Toxicol 1990, 28:72-78.

37. Hollander M, Wolfe DA: Nonparametric Statistical Methods. New York: Wiley; 1973.

38. Del Prato $S$, Felton A-M, Munro $N$, Nesto R, Zimmet P, Zinman B, on behalf of the Global Partnership for Effective Diabetes Management: Improving glucose management: ten steps to get more patients with type 2 diabetes to goal. Recommendations from the Global Partnership for Effective Diabetes Management. Int J Clin Pract 2005, 59:1345-1355.

39. Polli JW, Humphreys JE, Harmon KA, Webster LO, Reese MJ, MacLauchlin CC: Assessment of remogliflozin etabonate, a sodium-dependent glucose co-transporter-2 inhibitor, as a perpetrator of clinical drug interactions: a study on drug transporters and metabolic enzymes. J Diabetes Metab 2012, 3:5. http://dx.doi.org/10.4172/2155-6156.1000200.

\section{doi:10.1186/2050-6511-14-25}

Cite this article as: Hussey et al:: Safety, pharmacokinetics and pharmacodynamics of remogliflozin etabonate, a novel SGLT2 inhibitor, and metformin when co-administered in subjects with type 2 diabetes mellitus. BMC Pharmacology and Toxicology 2013 14:25. 